\title{
Polar Ring Galaxies and the Tully Fisher relation: implications for the dark halo shape
}

\author{
E. Iodice, M. Arnaboldi \\ INAF - Osservatorio Astronomico di Capodimonte, via Moiariello 16, 80131 Napoli, Italy \\ iodice@na.astro.it, magda@na.astro.it \\ F. Bournaud, F. Combes \\ Observatoire de Paris, LERMA, 61 Av. de l'Observatoire, F-75014, Paris, France \\ frederic.bournaud@ens.fr, Francoise.Combes@obspm.fr \\ L. S. Sparke \\ University of Wisconsin, Department of Astronomy, 475 N. Charter St., Madison, WI \\ 53706-1582, U.S.A. \\ sparke@astro.wisc.edu \\ W. van Driel \\ Observatoire de Paris, Section de Meudon, GEPI, CNRS FRE 2459, 5 place Jules \\ Janssen, F-92195 Meudon Cedex, France \\ Wim.vanDriel@obspm.fr \\ M. Capaccioli \\ INAF - Osservatorio Astronomico di Capodimonte, via Moiariello 16, 80131 Napoli, Italy \\ capaccioli@na.astro.it
}

\begin{abstract}
We have investigated the Tully-Fisher relation for Polar Ring Galaxies (PRGs), based on near infrared, optical and H I data available for a sample of these peculiar objects. The total K-band luminosity, which mainly comes from the central host galaxy, and the measured H l linewidth at $20 \%$ of the peak line flux density, which traces the potential in the polar plane, place most polar rings of the sample far from the Tully-Fisher relation defined for spiral galaxies, with
\end{abstract}


many PRGs showing larger $\mathrm{H}$ I linewidths than expected for the observed $\mathrm{K}$ band luminosity. This result is confirmed by a larger sample of objects, based on Bband data. This observational evidence may be related to the dark halo shape and orientation in these systems, which we study by numerical modeling of PRG formation and dynamics: the larger rotation velocities observed in PRGs can be explained by a flattened polar halo, aligned with the polar ring.

Subject headings: galaxies: peculiar - galaxies: kinematics and dynamics

\section{Introduction}

Polar Ring Galaxies (PRGs) are peculiar objects composed of a central spheroidal component, the host galaxy, surrounded by an outer ring, made up of gas, stars and dust, which orbits nearly perpendicular to the plane of the gas-poor central galaxy (Whitmore et al. 1990). Previous papers (Arnaboldi et al. 1995; Arnaboldi et al. 1997, Iodice et al. 2002a, 2002b, 2002c) found that even where the morphology of the host galaxy resembles that of an early-type system, PRGs show many similarities with late-type galaxies. The PRGs are characterised by a large amount of neutral hydrogen (H I), always associated with the polar structure (Schechter et al. 1984; van Gorkom et al. 1987; Arnaboldi et al. 1997), and by a gas-to-total luminosity ratio in the B-band typical of late-type galaxies.

The connection between PRGs and spirals is important for two of the best studied systems: NGC 660 (van Driel et al. 1995) and NGC 4650A, for which the new surface photometry (Iodice et al. 2002a; Gallagher et al. 2002), based on near-infrared (NIR) and optical Hubble Space Telescope (HST) data, have confirmed that the polar structure appears to be a disk of a very young age. By exploring the properties of the host galaxy and ring in the optical and NIR, for a sample of PRGs, Iodice et al. (2002a, 2002b, 2002c) found that the connection with spirals is tighter. Iodice and collaborators found that in almost all PRGs $i$ ) the host galaxy is bluer (even NIR and optical colors are similar to those of late-type galaxies) and younger (age vary between 1 and 5 Gyrs) than normal early-type galaxies, and it is characterised by a "compact", nearly-exponential bulge, with a brighter and smaller disk than those found in standard S0 galaxies; ii) the polar structure is even bluer than the host galaxy, and it has colors similar to those of dwarf irregular and spiral galaxies.

The Tully-Fisher relation (TF) is the most important scaling relation for disks (Tully \& Fisher, 1977): this is an empirical relationship between the disk rotational velocity $\left(V_{\text {rot }}\right)$ and its absolute luminosity $(L)$, where $L \propto V_{\text {rot }}^{4}$, approximately. The TF is extensively used to 
estimate extragalactic distances (e.g. Sakai et al. 2000; Tully \& Pierce 2000, and references therein). Furthermore, this relation is considered a critical constraint in galaxy formation theories (Dalcanton et al. 1997; McGaugh \& de Blok 1998; Mo et al. 1998; Steinmetz \& Navarro 1999, van den Bosch 2000). Several studies have addressed the origin of the TF relation (Kauffmann, White \& Guiderdoni 1993; Cole et al. 1994; Silk 1997; Avila-Reese, Firmani \& Hernandez 1998, Heavens \& Jiminez 1999; Elizondo et al. 1999; van den Bosch, 2000), but a definitive conclusion is yet to be found.

In the past few years, several studies have asserted the validity of the TF relation for some classes of disk galaxies which show different photometric and kinematical properties with respect to 'classical', high-surface-brightness spiral galaxies (Matthews, van Driel \& Gallagher 1998a, 1998b; McGaugh et al. 2000; Chung et al. 2002). In particular, the studies by Matthews et al. (1998b) and McGaugh et al. (2000), while exploring the TF relation for extreme late-type spiral galaxies and for faint field galaxies, indicated the validity of the "baryonic TF relation", which accounts for the gas mass in addition to the mass of the optical component by making a baryonic correction (Milgrom \& Braun 1988). These latest developments indicate that the TF relation is probing a very close liaison between the dark halo parameters and the total quantity of baryons in galaxies: the dark halo, which is responsible for the $\mathrm{H}$ I linewidth and the flat rotation curve in the outer regions of a disk, is tuned to the total amount of baryons in the luminous component.

In PRGs, the H I linewidth $(\Delta V)$ measures the dynamics along the meridian plane, which is dominated by the dark matter, while the baryons are nearly equally distributed between the host galaxy and the polar ring. We wish to investigate the position of the PRGs in the $\log (\Delta V)-L$ plane, and study via N-body simulations of 3-D systems whether the dark halo shape may influence their position in the $\log (\Delta V)-L$ plane, with respect to the TF relation of bright disks. The question of the dark halo shape is important $i$ ) to constrain dark matter models, through cosmological simulations (Navarro, Frenk \& White 1996, 1997; Bullock et al. 2001) which predict the distribution of the halo shapes and the universal radial dependence of the dark matter distribution; ii) to give hints on the nature of dark matter (see Combes 2002 as a review); and furthermore iii) the dark halo properties in PRGs can give important constraints on the formation scenarios for these peculiar objects, which is still an open issue (see Iodice et al. 2002a and 2002c and references therein).

In Section 2 we present the NIR, optical and HI observations available for a sample of PRGs and discuss the PRGs location in the $\log (\Delta V)-L$ plane; and we derive some insights on the dark halo shape in these objects via N-body, 3- and 2-D models in Section 3 . In Section 4 we evaluate the uncertainties and discuss our findings with respect to relevant dark halo parameters, i.e. total dark mass, central density and flattening, and then derive 
our conclusions in Section 5.

\section{Observations}

\subsection{Near-infrared, $\mathrm{B}$ band, and $\mathrm{H}_{\mathrm{I}}$ observations of polar ring galaxies}

New near-infrared J, H and Kn images are available for a sample of PRGs (Iodice et al. 2002a, 2002b, 2002c), which are selected from the Polar Ring Catalogue (Whitmore et al. 1990), and for ESO 235-G58, which was classified as a PRG related object by Buta \& Crocker (1993). Data were obtained at the $2.3 \mathrm{~m}$ telescope of the Mt. Stromlo and Siding Spring Observatory, with the CASPIR infrared camera (McGregor 1994). The angular resolution of this camera is 0.5 arcsec pixel $^{-1}$ and it has a field of view of $2.0^{\prime} \times 2.0^{\prime}$. A detailed description of the data reduction and analysis is given by Iodice et al. (2002a, 2002b, 2002c). The average photometric error on $\mathrm{K}$ magnitudes is $0.05 \mathrm{mag}$, and a detailed discussion is given in Iodice et al. (2002b). This sample contains only 6 PRGs, while more data are available in the optical bands. Since we wish to investigate the validity of the TF relation for PRGs, it is worthwhile to compare the PRG properties with those of a very large sample of spiral galaxies. To derive the TF relation for normal disk galaxies, we will use the very large and detailed dataset available in the I-band from Giovanelli et al. (1997). The B band magnitude is known for many PRGs (Whitmore et al. 1990; Van Driel et al. 2000a, 2002b; Gallagher et al. 2002), and we estimate the B band magnitude for spiral disks in the sample from Giovanelli et al. using the observational relation between morphological type index and the $B-I$ colors (de Jong 1996).

In PRGs the bulk of the light comes from the host galaxy, which is a nearly dust free component in most systems (see Iodice et al. 2002a, 2002b). In most PRGs of our sample, the central most luminous part of the host galaxy is not obscured by the dust in the PR region which passes in front of it, so there is no significant correction for internal extinction. For the 3 PRGs (ESO 603-G21, ARP 230, ESO 235-G58) where the dust associated with the PR obscures the central regions of the host galaxy, we have corrected the $\mathrm{K}$ and $\mathrm{B}$ total magnitudes for internal extinction, following the equations from Tully et al. (1998). We expect little extinction within the polar ring itself, since this component resembles low-

surface-brightness disk galaxies (see the HST images in Iodice et al. 2002a and Gallagher et al. 2002), where dust extinction is not high (Matthews and Wood 2001).

The Hi integrated line profile data were obtained from several published hydrogen observations of PRGs, carried out by e.g. Richter et al. (1994), van Gorkom et al. (1987), van Driel and collaborators (2000, 2002a, 2002b), with several radio telescopes. 
The absolute Kn magnitude $\left(M_{K n}\right)$, B band absolute magnitude, and measured linewidth at $20 \%$ of the peak line flux density $\left(\Delta V_{20}\right)$, for each PRG in our sample are listed in Table 1. For all distance-dependent quantities we have assumed $H_{0}=75 \mathrm{~km} \mathrm{~s}^{-1} \mathrm{Mpc}^{-1}$.

\subsection{PRGs and the TF relation for spiral galaxies}

We wish to compare the position of the PRGs in the $\log (\Delta V)-L$ plane with the $\mathrm{TF}$ relation observed for bright spiral galaxies. In principle, we do not know what expect, because the two quantities, rotational velocity and absolute luminosity, are related to two different components in the PRG, the polar structure and the host galaxy respectively, which lie in two decoupled planes. In fact the total $\mathrm{K}$ light comes mostly from the central galaxy (Iodice et al. 2002a, 2002b, 2002c), whereas the observed H I linewidth traces the dynamics of the polar structure along the host galaxy's meridian plane.

In Figure 1 we show the K-band TF relation for a sample of spiral galaxies studied by Verheijen $(1997,2001)$. The values of $M_{K}$ and $\log \left(\Delta V_{20}\right)$ for PRGs are also shown on this

plot. Our and Verheijen's data sets have very similar photometric properties and limiting magnitudes.

We see that five PRGs lie near to the high-velocity boundary of the TF relation, or show larger velocities (for a given luminosity) than disk galaxies. Only the PRG AM2020-504 shows a lower rotation velocity for its K-band absolute magnitude.

In the B-band, the tendency of PRGs to have larger velocities with respect to the TF is confirmed for a larger sample of PRGs compared with data for 787 disk galaxies (Giovanelli et al. 1997). In Figure 2, we see that two PRGs lie at lower velocities than those predicted by the TF for spiral galaxies, two objects lie on the TF relation, and twelve PRGs either lie on the high velocity boundary of the TF relation or show much larger velocities. Van Driel et al. (2002b) also find that most kinematically confirmed PRGs show larger H I profile widths than bright spiral galaxies, at a given luminosity.

\section{The PRG positions in the $\log (\Delta V)-L$ plane and their implications for the dark halo}

Observing higher or lower velocities with respect to the linear TF of disk galaxies is relevant for the discussion on the dark halo shape. Via analytical models and simple assumptions about the mass distribution, either luminous or dark, we can estimate where the 
PRGs ought to lie in the $\log (\Delta V)-M_{K / B}$ plane (Fig.1 and Fig.2), with respect to the TF relation for disk galaxies. If there were no dark matter, and the gravitational potential were oblate in the same sense as the flattened host galaxy, the polar ring would acquire an eccentric shape. When the polar ring and the host galaxy are both seen edge-on, which is close to being the case for most of our PRGs, the net effect will be that the line-of-sight (LOS) polar ring velocities are reduced, see Fig. 3. In the logarithmic, scale-free, potential case, a simple formula gives the expected velocity ratio between the major and the minor axis components as:

$$
\epsilon_{v}=1-\frac{v_{\text {major }}}{v_{\text {minor }}}=\epsilon_{\rho} \simeq 2 \epsilon_{\Phi}
$$

from Gerhard \& Vietri (1986), where $\epsilon_{\rho}$ is the flattening (1-axis ratio) of the density distribution and $\epsilon_{\phi}$ is the potential flattening. Therefore we would expect PRGs to have on average lower velocities with respect to what would be measured in the equatorial plane: see Fig.4. This implies that when the polar structure is eccentric, the observed LOS velocities in $\Delta V_{20}$ are the smallest, i.e. those from the particles in the polar regions, as shown in Figure 4. Thus the observed $\Delta V_{20}$ depends on both the mean velocity along the ring, and the ring eccentricity. On the contrary, Fig.1 and Fig.2 show that the majority of PRGs have larger velocities than expected in the $\log (\Delta V)-M_{K / B}$ plane. Therefore we need to investigate how these velocities can be produced, and how they may depend on the intrinsic properties of the dark galaxy halo.

We first compute a series of N-body models of the formation of polar rings in galaxy mergers or by tidal accretion of gas from another galaxy (Bournaud \& Combes 2002). Both scenarios are known to form polar structures (see Reshetnikov \& Sotnikova 1997; Bekki 1997, 1998). The numerical code computes the gravitational potential via an FFT method (James 1977), and includes sticky particles gas dynamics (Schwarz 1981), and star formation that is assumed to obey a generalised Schmidt law (Schmidt 1959). Both scenarios for formation of polar rings give similar results for the kinematics of the ring after its formation. The details of the N-body models and numerical codes are described in Bournaud \& Combes (2002). Fig. 5 shows an example of the structures produced.

These 3D N-body models do not allow us to choose the dark halo shape after the polar ring is formed. Moreover, we cannot separate the influence of the host galaxy potential, the dark halo flattening, and the ring self-gravity, which are all important in order to understand what our observations may imply for the PRG structure. We therefore developed a simpler model, where every parameter can be varied. In this model, the dark halo and the host galaxy are two rigid potentials. The eccentricity of the polar structure is given by the shape of the particle orbits in this rigid potential. Particles are launched around the polar plane and are followed for 4 Gyrs, before their kinematics is analysed. The polar ring self-gravity 
may also be included via a 2-D FFT algorithm that computes the gravitational potential in the polar plane.

Our N-body 3D models of polar rings (see Figure 5) show different characteristics depending on the shape of their dark halo. We see that when the halo is oblate and flattened towards the host galaxy, the observed velocities are then smaller, and the PRGs lie on the left side of the TF relation of high-surface-brightness disks. When the oblate halo is flattened towards the polar ring, the observed velocities are larger, shifting the PRGs to the right side of the diagram. As most of the observed PRGs have larger velocities compared to the TF relation for spiral galaxies, it seems to suggest that their dark halo is flattened towards the polar plane.

The second model is initially computed for massless rings. We choose a $10 \mathrm{kpc}$-scaled host galaxy, and a polar ring whose mean radius is $15 \mathrm{kpc}$. The dark halo is computed to produce a flat rotation curve at large radii, its mass is about 0.8 times the host mass inside $10 \mathrm{kpc}$ radius, and 1.4 times the host galaxy mass inside $15 \mathrm{kpc}$. A halo flattened towards the equatorial plane reduces the measured spread in radial velocity in the polar structure: the more flattened the potential, the more eccentric the ring is and the smaller the observed velocities are. When the halo is spherical, the potential is flattened by the host galaxy luminous mass, therefore the ring is still eccentric, and the PRG velocities then lie on the low-velocity boundary of the TF relation, far from the observational result. This is what was found by Combes and Arnaboldi (1996) in their detailed model for the polar ring galaxy NGC4650A: a spherical halo cannot reproduce the large ring velocities, because the ring is eccentric, unless when the halo is very massive (but this case is easily ruled out, see Sect. 4.4).

We now wish to account for the ring self-gravity: the ring mass for all objects in our sample is believed to be a significant fraction of the stellar mass of the host galaxy or of the same order, as it is in the case for NGC 4650A (Iodice et al. 2002a). When the halo is spherical or flattened towards the host galaxy equatorial plane, its self-gravity makes the polar ring more circular (see Figure 6). In Figure 1 we have shown the effects for polar rings whose mass is equal to the mass of the host galaxy. When the polar ring is lighter, these effects are smaller; for instance when the polar mass is half the mass of the luminous HG, we find results that are comparable to those obtained with the first model. When self-gravity is considered, the simulated PRGs lie near the TF relation for bright disk systems of Fig.1. And yet, when the flattened dark halo is aligned with the host galaxy equatorial plane, the ring's self-gravity cannot account for the large velocities observed in most polar rings, even when the ring is as massive as the host galaxy. But, when dark halos are flattened towards the polar structures, the ring self-gravity is less important, because the flattened potential 
already makes rings more circular: the positions of the corresponding points does not move much from the the massless ring case, when the ring mass is increased, see Figure 1. Indeed such systems seem able to explain the large velocities observed in the polar structures, and confirms the conclusions drawn from our 3-D accretion and merging models.

Let us now consider the case of AM 2020-504 and UGC 4261, which show lower velocities than bright disks, for their observed $\mathrm{K}$ and $\mathrm{B}$ band absolute magnitudes. Both galaxies have narrow rings with very small masses, therefore they are well described by our massless ring model. This model easily predicts the observed velocities in the polar ring, when the potential is significantly flattened towards the host galaxy equatorial plane. This is confirmed in the case of AM 2020-504 by the photometric study of the host galaxy, which is a flattened elliptical (E4, see Arnaboldi et al. 1993). Moreover, if those systems are embedded in massive dark halos that contribute much to the potential near the polar ring, these halos must be flattened too.

\section{Discussion}

In the $\log (\Delta V)-M_{K / B}$ planes shown in Fig. 1 and Fig. 2, most PRGs have larger H I rotation velocities than standard spiral galaxies, at a given $\mathrm{K}$ or B-band luminosity of the stellar component. Our N-body simulations have suggested that a likely explanation for this effect is a flat dark halo, whose main plane is aligned with the host galaxy meridian plane, and prevents the polar ring to become eccentric. The question arises if other effects, i.e. non-homogeneities in the TF relations for spirals, caused by bar and/or non edge on disks, or larger $\mathrm{M} / \mathrm{L}$ ratios, can produce similar results and therefore be alternative explanations for the high velocities observed in PRGs.

\subsection{Possible biases in the spiral sample}

PRGs are peculiar objects that are easily identified when both their central component and polar ring are observed nearly edge-on. When dealing with the TF relation for spiral galaxies, we have assumed that it was valid for axisymmetric edge-on disks. Yet, the large sample from Giovanelli et al. (1997) contains barred disks and disks at lower inclination with respect to the LOS. Bars may cause a bias in the observed velocities, and calculating the real value of $\Delta V$ for a disk at a lower inclination cannot be done exactly. We have therefore selected from the Giovanelli et al. sample those disk galaxies that are not barred and are seen nearly edge-on $(i \geq 80)$; we built a new TF relation from this new sample of 
179 disks, which is now free from those biases that may cause lower H I velocities. The linear interpolation of this new TF relation is shown in Figure 2 : it is still very close to the one of the original sample and indicates that barred disks and disks with lower inclination do not modify the mean TF relation (although the scatter is smaller, for bars increase the scatter of the TF relation). So this cannot explain why PRGs have larger observed H I velocities than bright disks.

\subsection{The baryonic Tully-Fisher relation}

We have already mentioned (Sec. 1) that PRGs are gas-rich, thus we wish to investigate whether once we account for the gas mass, this might move them back on the TF relation, i.e. if they are close to the baryonic TF relation (Matthews et al. 1998b; McGaugh et al. 2000). A mass-to-velocity TF relation for PRGs is shown in Figure 7: PRGs are now closer to the TF relation for disk galaxies, but still do not lie on this relation: i.e. the higher velocities in the PR cannot be explain by additional mass in the $\mathrm{H}$ I associated within the PR itself.

\subsection{Host galaxy mass-over-light ratios}

Another possible explanation for higher $\mathrm{H}$ I rotation velocities is that PRGs have larger mass-to-light ratios than spiral disks of similar K\&B-band luminosity, i.e. for the same observed $\Delta V_{20}$, PRGs may be less luminous on average in $\mathrm{K} / \mathrm{B}$ than standard spiral disks. Therefore we have computed the linear interpolation of the TF relation for the $S a$ galaxies alone, of the Giovanelli et al. (1997) sample. The average B-band magnitude of this sample corresponding to $\log (\Delta V)=2.5$ is -19.35 , while it is brighter, -19.16 , for the whole Giovanelli sample. Still, the difference of 0.19 mags is not enough to place most PRGs on the TF relation, which would require a value 5 times larger.

\subsubsection{Comparison between PRG host galaxies and SOs}

The similarities between PRGs and spiral galaxies, from NIR and optical photometry, prompted us to compare PRGs with the TF for early-type spiral galaxies rather than with lenticulars. But we have also compared the position of PRGs in the $\log (\Delta V)-M_{B}$ plane 
with the TF relation for S0 galaxies ${ }^{1}$ (Neistein et al. 1999, Mathieu et al. 2002), and we find that many PRGs lie near the TF relation for S0 galaxies. The offset between the TF relation for $\mathrm{S} 0$ galaxies and the TF for bright spiral galaxies is caused by the S0s being less luminous than bright spirals, at a given velocity. As suggested by Mathieu et al. (2002), this is consistent with the formation scenario in which S0 galaxies were formed from ordinary late-type spirals which were stripped of their star-forming medium.

Can this be a valid explanation for the PRGs position in the $\log (\Delta V)-M_{K / B}$ plane? If the PRG positions in this plane were caused by larger M/L ratios for the luminous component, as is the case for lenticular galaxies, it must be so for the host galaxy too, which is the component most similar to an $\mathrm{S} 0$ galaxy. We have computed $\log (\Delta V)$ for the host galaxies of the best studied systems, NGC 4650A, NGC 660, NGC 2685, UGC 7576 and A0136-0801, (Sackett et al. 1994; van Driel et al. 1995; Simien \& Prugniel 1997; Whitmore et al. 1990; Schweizer et al. 1983), using optical absorption-line rotation curves along the host galaxy equatorial plane; these are shown in Fig. 8. The host galaxies in these five systems fall on the TF relation of bright spiral disks, which indicates that $i$ ) the mass-to-light ratio of this component is different from those of standard S0s, as also found in recent studies of PRG NIR and optical colors (see Iodice et al. 2002a, 2002b, 2002c) and ii) a luminous-to-dark matter content similar to those of standard bright disks.

\subsection{Peculiar properties of the dark halo}

Gerhard et al. (2001) showed that elliptical galaxies follow a TF relation in the $\log (\Delta V)-$ $M_{*}$, where $M_{*}$ is the total mass in the luminous component, which is shallower than the relation for spiral galaxies, even when the maximal $M / L_{B}$ is adopted to compute the total stellar masses. This led Gerhard and collaborators (2001) to infer that elliptical galaxies have slightly lower baryonic mass than spiral galaxies of the same circular velocities, and that their dark halos are denser than halos of spiral galaxy with the same $L_{B}$. How much more massive must the dark halo be, to account for the velocities observed in polar rings? In our numerical models, we had assumed a dark-to-visible mass ratio of about 1 inside the optical radius ${ }^{2}$, as it is the case in spiral galaxies, and only flattened polar halos could reproduce the observed $\mathrm{HI}$ velocities. The observed value of $\Delta V_{20}$ depends largely on the dark halo shape and the ring eccentricity, while it varies only as the square root of the total

\footnotetext{
${ }^{1}$ The TF relation for S0 galaxies was obtained by comparing the asyntotic rotation speeds of stars with the absolute magnitude (see Mathieu et al. 2002).

${ }^{2}$ For PRGs, the optical radius correspond to the ring average extension.
} 
mass (and depends even less on the dark mass). Thus, a large amount of dark matter is needed when the halo is not polar: if one wishes to reproduce the PRG position on the TF diagram with a spherical halo, then the dark mass needed is twice than the visible mass. With an E5 equatorial halo, the dark-to-visible mass ratio raises to about 3.5.

An increased total dark halo mass alone can explain the observed position of PRGs on the $\log (\Delta V)-M_{B}$ plane, but the required large dark masses are unrealistic: the fact that the PRGs host galaxies lie within the TF for spiral galaxies (see Fig. 8) indicates that the dark matter content in PRGs is similar to that of bright spiral galaxies.

Also in the spherical halo case, the host galaxy would be offset too from the TF relation of bright disks, because the dark mass. In the case of NGC 4650A, Combes \& Arnaboldi (1996) found that no dark matter was required to reproduce the host kinematics, and that the dark-to-visible mass ratio could not be as large as required by a spherical halo. If we want to account for observed velocities in PRGs with a spherical halo, we have to believe that the halo density drops more slowly than $1 / r$.

A prolate dark halo associated with the host galaxy, i.e. with the major axis aligned with the HG major axis, could also account for the large velocities observed in rings, provided it is massive enough. To reproduce the large velocities in the PR, such a halo must be even more massive than for the oblate case. For an E5 prolate halo and a ring mass that amounts to $25 \%$ of the visible mass, we find that the dark-to-visible mass ratio inside the optical radius would be close to 4 . Such a massive halo would cause a large offset of the host galaxy from the TF relation of bright disks, which is not observed. On the other hand, a prolate halo with a polar major axis can account for the velocities in PRGs and this is one of the possibility of a halo flattened towards the polar ring.

Given the constraint from the host galaxy observed kinematics, the dark halo shape alone can explain the high rotation velocities observed in polar structures, provided that the short axis of the halo lies perpendicular to the polar ring, in the equatorial plane of the central galaxy. In the case of NGC 4650A, Combes \& Arnaboldi (1996) had already argued that a dark halo flattened towards the polar ring required less dark mass to account for the large velocities observed in the polar ring, while the visible mass alone was enough to account for the velocities in the host galaxy.

\section{Conclusion}

In this paper we have explored the position of PRGs in the $\log (\Delta V)-M_{K / B}$ plane with respect to the TF relation for spiral galaxies. The majority of PRGs show larger 
rotation velocities at a given luminosity than spiral galaxies. A comparison with the N-body simulations has led us to conclude that these large velocities indicate that the dark halo is most likely flattened towards the polar ring plane. This explains the position of PRGs with respect to the TF relation for normal spiral disks, the position of the host galaxy within the TF of bright disks, and it may become an important constraint on the nature of dark matter.

The 3-D simulations in this work were computed on the Fujitsu NEC-SX5 of the CNRS computing center, at IDRIS. This research made use of the NASA/IPAC Extragalactic Database (NED) which is operated by the Jet Propulsion Laboratory, California Institute of Technology, under contract with the National Aeronautics and Space Administration. M.A. wishes to thank O. Gerhard for many useful discussions on the TF relation for elliptical galaxies and for his comments on an earlier version of this paper.

\section{REFERENCES}

Arnaboldi, M., Capaccioli, L., Cappellaro, E., Held, E. V. \& Sparke, L. S. 1993, A\&A, 267, 21

Arnaboldi, M., Freeman, K. C., Sackett, P. D., Sparke, L. S. \& Capaccioli, M. 1995, Planet. Space Sci., 43, 1377

Arnaboldi, M., Oosterloo, T., Combes, F., Freeman, K. C. \& Koribalski, B. 1997, AJ, 113, 585

Avila-Reese, V., Firmani, C. \& Hernandez, X. 1998, ApJ, 505, 37

Bekki, K. 1997, ApJ, 490, L37

Bekki, K. 1998, ApJ, 499, 635

Bournaud, F. \& Combes, F. 2002, A\&A, in prep.

Bullock, J. S., Kolatt, T. S., Sigad, Y., Somerville, R. S., Kravtsov, A. V., Klypin, A. A., Primack, J. R. \& Dekel, A. 2001, MNRAS, 321, 559

Buta, R. \& Crocker, D. A. 1993, AJ, 106, 939

Chung, A., van Gorkom, J.H., O’Neil, K. \& Bothun, G.D. 2002, AJ, 123, 2387 
Cole, S., Aragon-Salamanca, A., Frenk, C. S., Navarro, J. F. \& Zepf, S. E. 1994, MNRAS, 271,781

Combes, F. \& Arnaboldi, M. 1996, A\&A, 305, 763

Combes, F. 2002, NewA Reviews, submitted (astro-ph/ 0206126)

Dalcanton, J. J., Spergel, D. N. \& Summers, F. J. 1997, ApJ, 482, 659

de Jong, R. S. 1996, A\&A, 313, 377

Elizondo, D., Yepes, G., Kates, R., Muller, V. \& Klypin, A. 1999, ApJ, 515, 525

Gallagher, J. S., Sparke, L. S., Matthews, L. D., Frattare, L. M., English, J., Kinney, A. L., Iodice, E. \& Arnaboldi, M. 2002, ApJ, 568, 199

Giovanelli, R., Haynes, M. P., Herter, T., Vogt, N. P., Wegner, G., Salzer, J. J., da Costa, L. N. \& Freudling, W. 1997, AJ, 113, 22

Gerhard, O. \& Vietri, M. 1986, MNRAS, 223, 377

Gerhard, O., Kronawitter, A., Saglia, R.P. \& Bender, R. 2001, AJ, 121, 1936

Heavens A. F. \& Jiminez, R. 1999, MNRAS, 305, 770

Iodice, E., Arnaboldi, M., De Lucia, G., Gallagher, J. S., Sparke, L. S. \& Freeman, K. C. 2002a, AJ, 123, 195

Iodice, E., Arnaboldi, M., Sparke, L. S., Gallagher, J. S., \& Freeman, K. C. 2002b, A\&A, 391, 103

Iodice, E., Arnaboldi, M., Sparke, L. S., \& Freeman, K. C. 2002c, A\&A, 391, 117

James, R. A. 1977, C. Comput. Phys., 25, 71

Kauffmann, G., White, S. D. M. \& Guiderdoni, B. 1993, MNRAS, 264, 201

Mathewson, D.S. \& Ford, V.L. 1996, ApJS, 107, 97

Matthews, L.D., van Driel, W. \& Gallagher, J.S., 1998a, AJ, 116, 1169

Matthews, L.D., van Driel, W. \& Gallagher, J.S., 1998b, AJ, 116, 2196

Matthews, L.D. \& Wood, K. 2001, ApJ, 548, 150

Mathieu A., M.R. Merrifield \& K. Kuijken 2002, MNRAS, 330, 251 
McGaugh, S. S. \& de Blok, W. J. 1998, ApJ, 499, 41

McGaugh, S. S., Schombert, J. M., Bothun, G. D. \& de Blok, W. J. 2000, ApJ, 533, 99

McGregor, P.J. 1994, PASP, 106, 508

Milgrom, M. \& Braun, E. 1988, ApJ, 334, 130

Mo, H. J., Mao, S. \& White, S. D. 1998, MNRAS, 295, 319

Navarro, J.F., Frenk, C.S. \& White, S.D. 1996, ApJ, 462, 563

Navarro, J.F., Frenk, C.S. \& White, S.D. 1997, ApJ, 490, 493

Neistein, E., Maoz, D., Rix, H.-W. \& Tonry, J. L. 1999, AJ, 117, 2666

Pfenniger, D., Combes, F. \& Martinet L. 1994, A\&A, 285, 79

Reshetnikov, V. \& Sotnikova, N. 1997, A\&A, 325, 933

Reshetnikov, V., Hagen-Thorn, V. A. \& Yakovleva, V. A. 1994, A\&A, 290, 693

Richter, O.-G., Sackett, P. D. \& Sparke, L. S. 1994, AJ, 107, 99

Sackett, P.D., Rix, H.-W., Jarvis, B.J., Freeman, K.C. 1994, ApJ, 436, 629

Sakai, S., Mould, J. R., Hughes, S. M., Huchra, J. P., Macri, L. M., Kennicutt, R. C., Jr., Gibson, B. K., Ferrarese, L., Freedman, W. L., Han, M., Ford, H. C., Graham, J. A., Illingworth, G. D., Kelson, D. D., Madore, B. F., Sebo, K., Silbermann, N. A. \& Stetson, P. B. 2000, ApJ, 529,698

Silk, J. 1997, ApJ, 481, 703

Schechter, P.L., Sancisi, R., van Woerden, H. \& Lynds, C.R. 1984, MNRAS, 210, 497

Schmidt, M. 1959, ApJ, 129, 234

Schwarz, M. P. 1981, ApJ, 247, 77

Schweizer, F., Whitmore, B.C. \& Rubin, V. 1983, AJ, 88, 909

Simien, F. \& Prugniel, Ph. 1997, A\&AS, 122, 521

Steinmetz, M. \& Navarro, J. F. 1999, ApJ, 513, 555

Tully, R. B. \& Fisher, J. R. 1977, A\&A, 54, 661 
Tully, R. B., Pierce, M. J., Huang, J.-S., Saunders, W., Verheijen, M. A. W. \& Witchalls, P. L. 1998, AJ, 115, 2264

Tully, R. B. \& Pierce, M. J. 2000, ApJ, 533, 744

van Driel, W., Combes, F. \& Casoli, F. et al. 1995, AJ,109, 942

van Driel, W., Arnaboldi, M., Combes, F. \& Sparke, L. S. 2000, A\&A, 141, 385

van Driel, W., Combes, F., Arnaboldi, M. \& Sparke, L. S. 2002a, A\&A, 386, 140

van Driel, W., Arnaboldi, M., Combes, F. \& Sparke, L. S. 2002b, A\&A, in prep

van Gorkom, J. H., Schechter, P. L. \& Kristian, J. 1987,ApJ, 314, 457

van den Bosch, F. C. 2000, ApJ, 530, 177

Verheijen, M. A. 1997, PhD thesis, Univ. Groningen, The Netherlands

Verheijen, M. A. 2001, ApJ, 563, 694

Whitmore, B. C., Lucas, R. A. \& McElroy, D. B. 1990, AJ, 100, 1489 


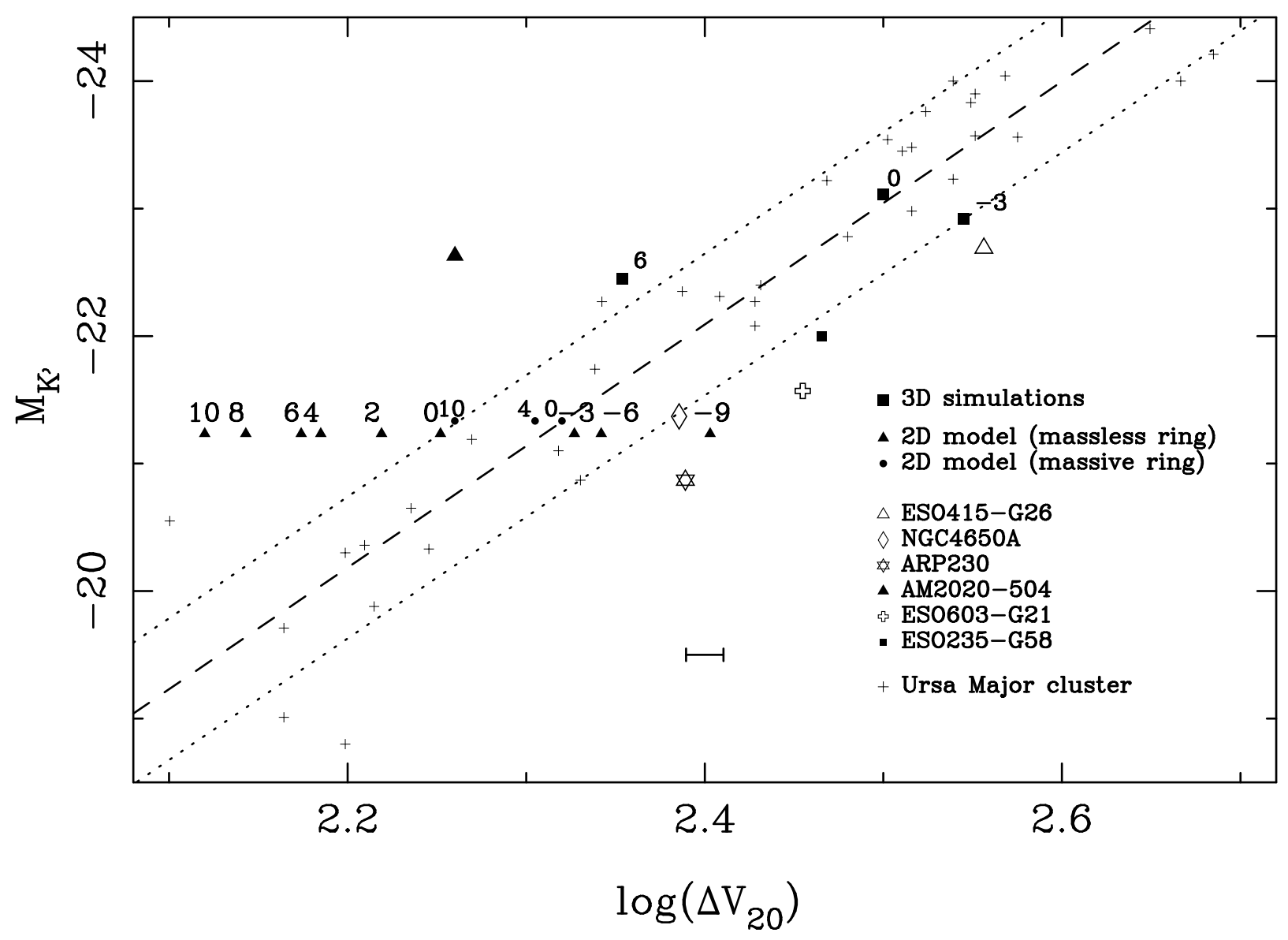

Fig. 1.- Absolute magnitude in the $\mathrm{K}$ band vs. the measured $\mathrm{H}$ I linewidth at $20 \%$ of the peak line flux density $\left(\Delta V_{20}\right)$, for PRGs of the selected sample, compared with a sample of spiral galaxies from Verheijen (2001), and with the results from 3-D simulations and 2$\mathrm{D}$ models (for massless rings and rings that are as massive as the host galaxy). We have assumed $H_{0}=75 \mathrm{~km} \mathrm{~s}^{-1} \mathrm{Mpc}^{-1}$, as made also by Verheijen. The long-dashed line is a linear interpolation of the TF relation for spiral galaxies, and the short-dashed lines show the width at $15 \%$ of the peak of the statistical distribution of spiral galaxies. For both models, the flattening of the halo is indicated next to each circle (massless ring) or triangle (very massive ring): a positive $x$ number indicates that it is an Ex halo with an equatorial flattening, while $-x$ corresponds to an Ex halo flattened toward the polar plane. The results from the 3-D models shown in this plot are those computed for the accretion scenario (Reshetnikov \& Sotnikova, 1997) ; our values for $\Delta V_{20}$ vs. $\mathrm{M}_{K}$ are very similar when one considers the merging scenario (Bekki, 1998). 


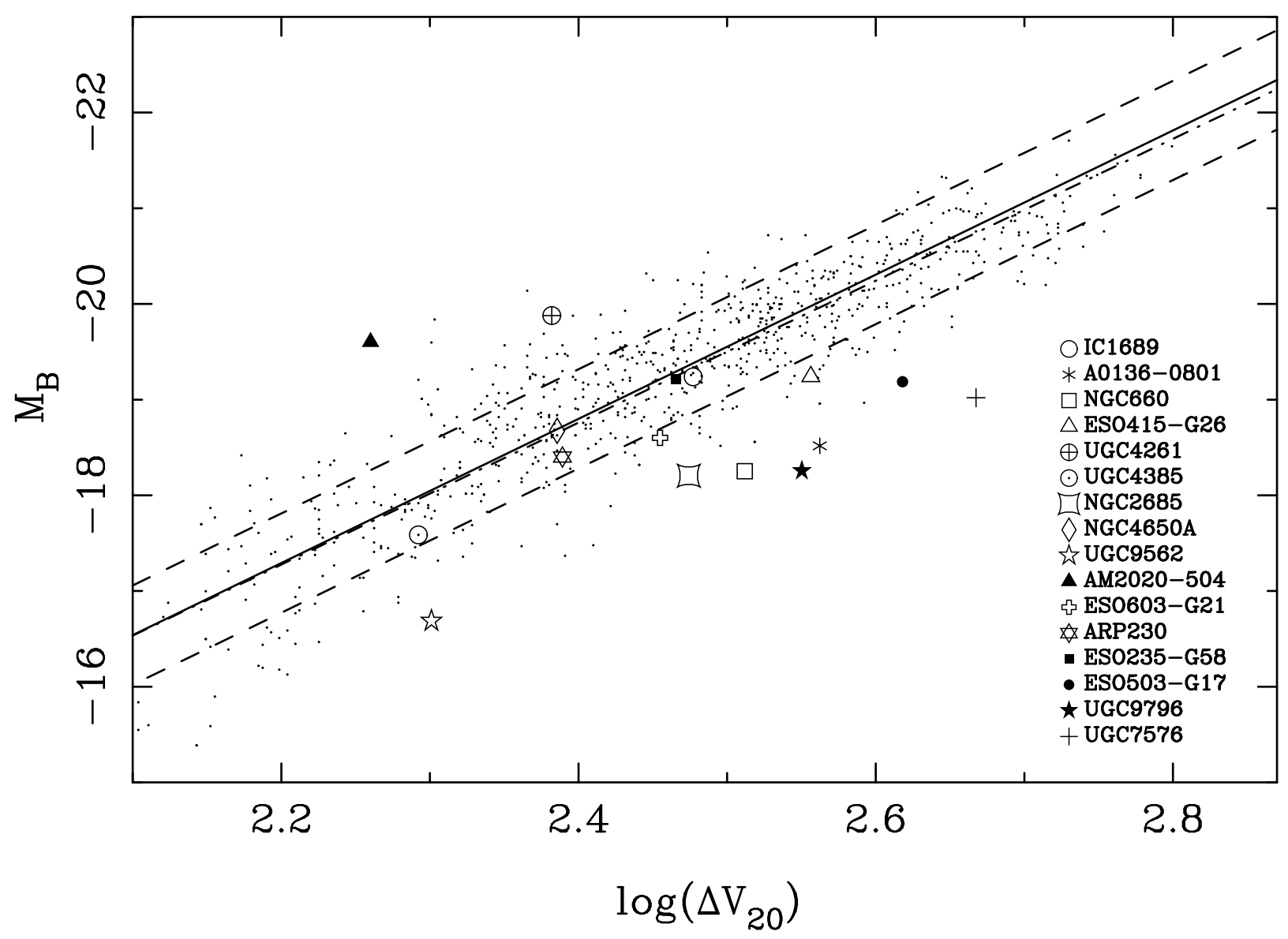

Fig. 2.- Absolute magnitude in the B band vs. the linewidth at $20 \%$ of the peak line flux density $\left(\Delta V_{20}\right)$, for 15 PRGs. Data for disk galaxies (dots) are from Giovanelli et al. (1997). Absolute magnitudes have been normalised to the same value of $H_{0}$ for PRGs and disk galaxies $\left(75 \mathrm{~km} \mathrm{~s}^{-1} \mathrm{Mpc}^{-1}\right)$. A linear interpolation of the TF relation is shown for these disk galaxies (solid line), $81 \%$ of which lie inside the dashed lines that are computed at $25 \%$ of the peak of the statistical distribution of spiral galaxies. The long-short dashed line is obtained for unbarred disks, seen nearly edge-on ( $i \geq 80$, see Section 4$)$. 


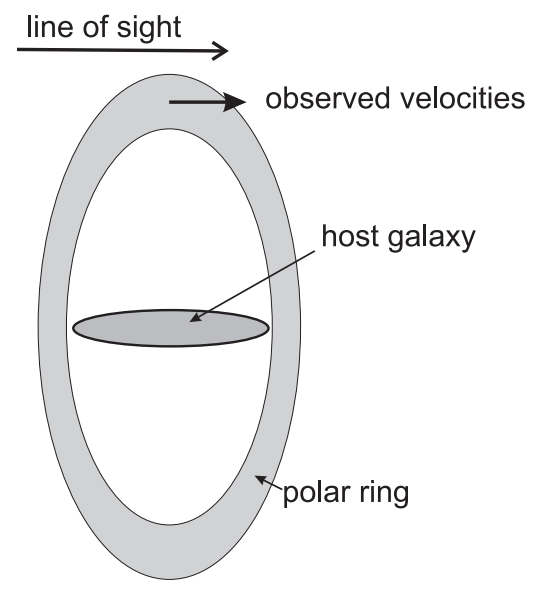

Fig. 3. - Line of sight for most PRGs: both components are seen nearly edge-on, thus the measured velocities in the polar structure are the smallest ones when the ring is eccentric, i.e. when the potential is flattened along the equatorial plane. This line of sight corresponds to the PRGs of our sample, and it is adopted for the analysis of the numerical models.

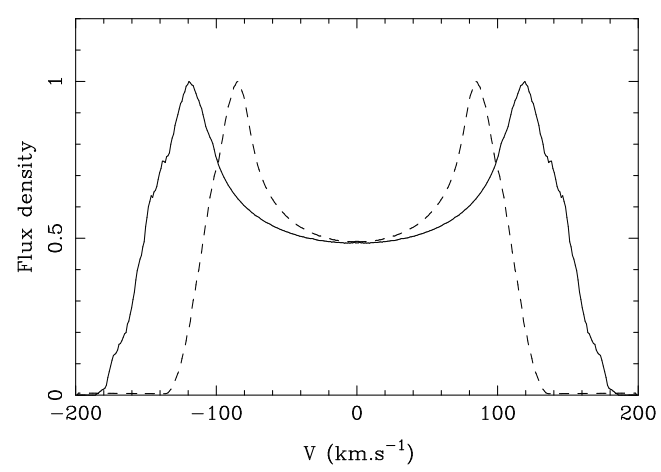

Fig. 4.- Simulated H I profiles for circular and eccentric polar rings seen edge-on, as shown in Figure 3. Solid line: H I profile computed for a circular ring, extending from 10 to 15 kpc. Dashed line: H I profile computed for an eccentric ring, with the same radial extension and radius, and ellipticity 0.35 (similar to the ring from 3-D simulations shown in Figure 5). For gas, we assume a velocity dispersion of $10 \mathrm{~km} / \mathrm{s}$. The values of $\log \left(\Delta V_{20}\right)$ are 2.51 for the circular ring and 2.38 for the eccentric ring. This difference is in agreement with the set of values for $\Delta V_{20}$, at a given total $\mathrm{K}$ or I band luminosity, obtained from PRG numerical models (see Figure 1). 

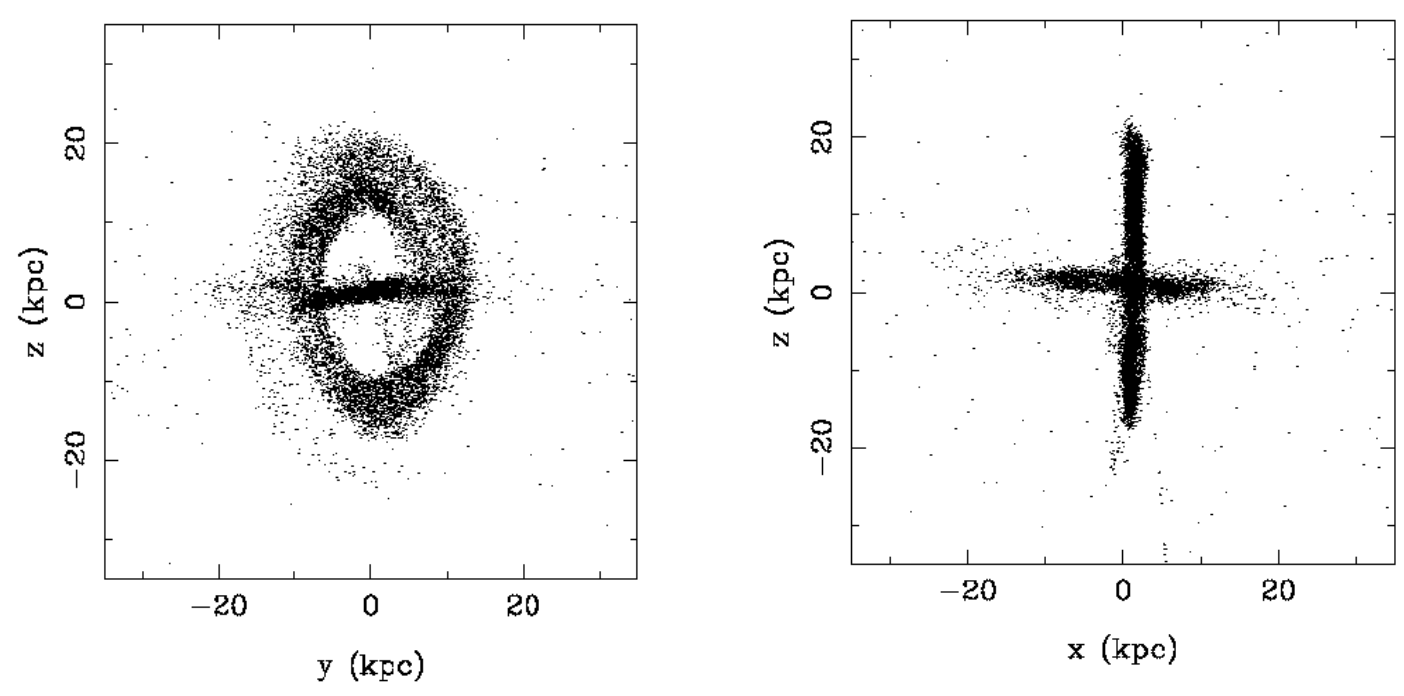

Fig. 5.- Example of polar ring formed in the 3-D N-body model, according to an accretion scenario. In most observed PRGs both the central disk and the polar ring are seen nearly edge-on (right panel). This polar ring is eccentric, since the gravitational potential is flattened by the host disk and an E4 dark halo. 

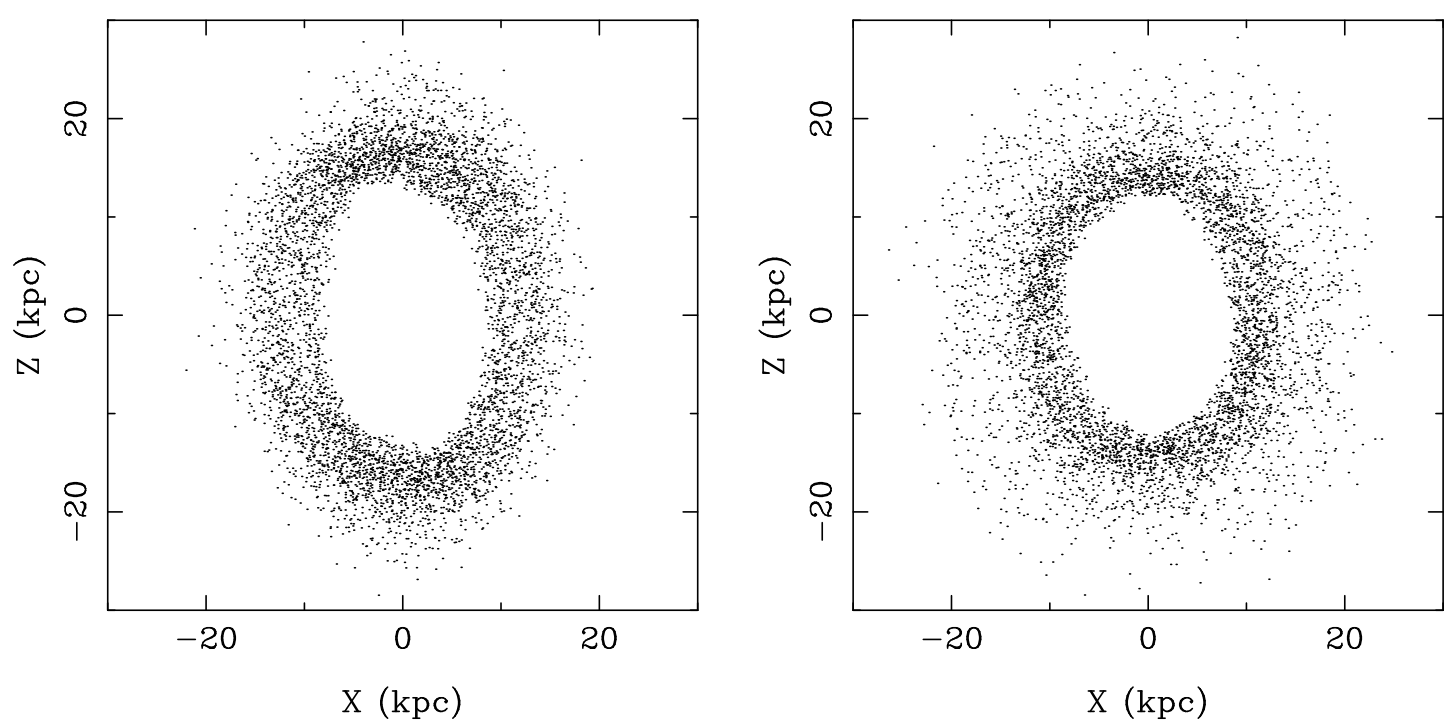

Fig. 6.- Polar rings from our 2-D model, formed during an accretion event, obtained with a E3 halo flattened in the equatorial plane $(x-y)$. The ring lies in the $z-x$ plane. The massless ring (on the left) is eccentric, which reduces the observed velocities. Though the very massive ring (on the right, the ring is as massive as the host galaxy) is rounder, its selfgravity is not sufficient to reproduce the observed velocities, unless the halo is also flattened towards the ring. The measured linewidth $\Delta V_{20}$ is $30 \%$ larger for the massive polar ring than for the massless ring.

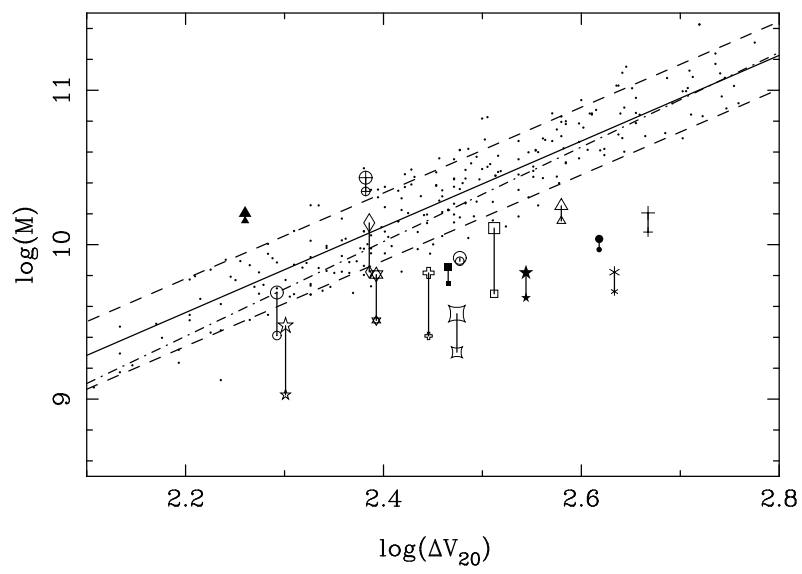

Fig. 7.- Plot of the stellar, mass in solar units, (smaller symbols for PRGs, long-short dashed line for spirals) and the stellar plus gaseous mass (solid line and dots for spirals, large symbols for PRGs) against the observed rotation velocities. Due to the similarities between host galaxies in PRGs and early-type spiral galaxies (Arnaboldi et al. 1995; Iodice et al. 2002a, 2002b), we have assumed $M / L=2$ for this component, as for spirals (de Jong 1996). In this plot, data for disk galaxies are from Mathewson \& Ford (1996). 


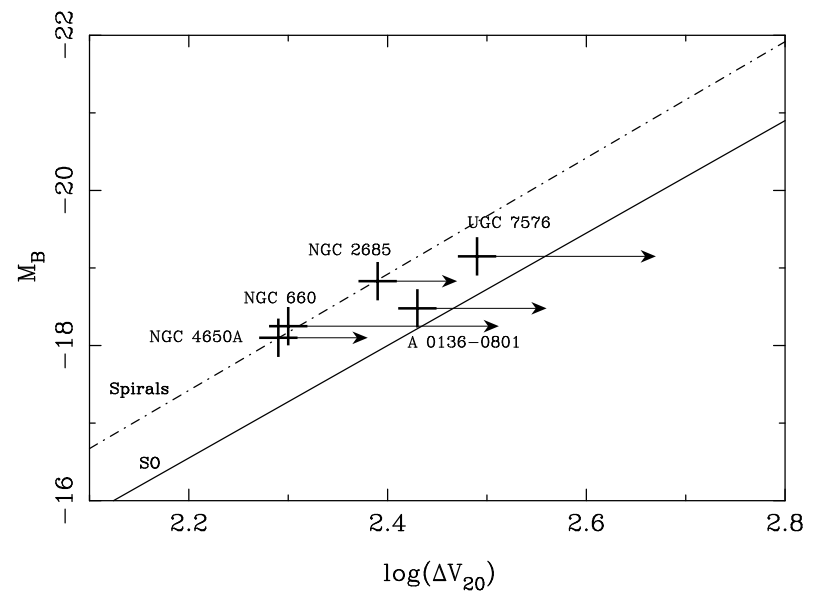

Fig. 8. - TF relation for S0 galaxies (data from Neistein et al. 1999 and Mathieu et al. 2002) and PRGs. The TF for spiral galaxies is plotted as a dashed line. Large crosses show the position of the central component in five PRGs. For NGC 4650A the circular velocity was derived from the rotation velocities and velocity dispersion of the stellar component (Combes \& Arnaboldi 1996), by computing the corresponding gas linewidth and assuming a $10 \mathrm{~km} / \mathrm{s}$ dispersion (which is the standard value for disks). The total B magnitude for NGC 4650A is from Iodice et al. 2002a. For NGC 660, velocities are derived from $H \alpha$ data (see van Driel et al. 1995). For NGC2586 and A0136-0801 the B magnitudes relative to the HG are given by Reshetnikov et al. 1994. The arrow shows the offset in $\log \left(\Delta V_{20}\right)$ between the velocities in the host galaxy equatorial plane and in the polar ring for each system. 
Table 1. Absolute Kn and B band magnitudes, heliocentric velocity, and H linewidth at $20 \%$ of the peak line flux density for a sample of polar ring galaxies.

\begin{tabular}{lcccccc}
\hline \hline Object name & PRC & $\begin{array}{c}V \\
\mathrm{~km} . \mathrm{s}^{-1}\end{array}$ & $\begin{array}{c}\mathrm{M}_{K n} \\
\mathrm{mag}\end{array}$ & $\begin{array}{c}\mathrm{M}_{B} \\
\mathrm{mag}\end{array}$ & $\begin{array}{c}\Delta V_{20} \\
\mathrm{~km} . \mathrm{s}^{-1}\end{array}$ & Ref. \\
\hline A0136-0801 & $\mathrm{A}-01$ & 5528 & & -19.23 & 365 & $\mathrm{vD}$ \\
ESO415-G26 & $\mathrm{A}-02$ & 4604 & -22.69 & -19.24 & 360 & $\mathrm{vD}$ \\
NGC2685 & $\mathrm{A}-03$ & 871 & & -18.20 & 335 & $\mathrm{vD}$ \\
UGC7576 & $\mathrm{A}-04$ & 7022 & & -19.02 & 465 & $\mathrm{vD}$ \\
NGC4650A & $\mathrm{A}-05$ & 2910 & -21.37 & -18.67 & 235 & $\mathrm{vD}$ \\
UGC9796 & $\mathrm{A}-06$ & 5420 & & -18.25 & 355 & $\mathrm{vD}$ \\
ARP230 & $\mathrm{B}-01$ & 1710 & -20.95 & -18.40 & 245 & $\mathrm{vD}$ \\
IC1689 & $\mathrm{B}-03$ & 4557 & & -20.40 & 300 & $\mathrm{vG}$ \\
ESO503-G17 & $\mathrm{B}-12$ & 10481 & & -19.19 & 415 & $\mathrm{vD}$ \\
UGC9562 & $\mathrm{B}-17$ & 1242 & & -16.68 & 200 & $\mathrm{vD}$ \\
AM2020-504 & $\mathrm{B}-19$ & 4963 & -22.63 & -19.60 & 182 & $\mathrm{vD}, \mathrm{A}$ \\
ESO603-G21 & $\mathrm{B}-21$ & 3124 & -21.74 & -18.60 & 285 & $\mathrm{vD}$ \\
NGC660 & $\mathrm{C}-13$ & 850 & & -18.25 & 325 & $\mathrm{vD}$ \\
UGC4261 & $\mathrm{C}-24$ & 6408 & & -19.87 & 240 & $\mathrm{vD}$ \\
UGC4385 & $\mathrm{C}-27$ & 1969 & & -17.58 & 195 & $\mathrm{vD}$ \\
ESO235-G58 & & 4262 & -22.13 & -19.21 & 292 & $\mathrm{vD}$ \\
\hline
\end{tabular}

a References for values of $\Delta V_{20}$ : telescope codes (V:VLA, N: Nançay, G: Green Bank, P: Parkes); vG: van Gorkom et al. (1987), vD: van Driel et al. (2000, 2002a, 2002b and references therein) and Richter et al. (1994), A: Arnaboldi et al. (1993).

${ }^{\mathrm{b}}$ The B band magnitude for NGC 4650A is from Gallagher et al. (2002). 